\title{
$\begin{array}{ll}\text { Ie portiQue } & \text { Le Portique } \\ \text { Revue de philosophie et de sciences humaines }\end{array}$ \\ e-Portique | 2006
}

\section{Soin, sacré, maternité}

Jean-Paul Resweber

\section{OpenEdition \\ Journals}

Édition électronique

URL : https://journals.openedition.org/leportique/898

DOI : $10.4000 /$ leportique.898

ISSN : $1777-5280$

\section{Éditeur}

Association "Les Amis du Portique"

\section{Référence électronique}

Jean-Paul Resweber, « Soin, sacré, maternité », Le Portique [En ligne], e-Portique, mis en ligne le 08 janvier 2007, consulté le 10 septembre 2022. URL : http://journals.openedition.org/leportique/898 ; DOI : https://doi.org/10.4000/leportique.898

Ce document a été généré automatiquement le 10 septembre 2022.

Tous droits réservés 


\title{
Soin, sacré, maternité
}

\author{
Jean-Paul Resweber
}

On a raison d'admirer les progrès de la science et des techniques au plan des pratiques concernant la grossesse et l'accouchement. Quand on pense que, au MoyenÂge, comme le montre $\mathrm{Ph}$. Ariès, plus de la moitié des enfants mouraient à la naissance. Mais le seul regard technique sur la grossesse, l'accouchement et les premiers jours suivant la naissance ne suffisent pas à énoncer l'insolite nouveauté que comporte l'événement d'une naissance. Les sciences et les techniques expliquent le processus de ces phénomènes, c'est-à-dire définissent des règles et des lois, mais l'explication n'est pas tout Ces phénomènes sont des phénomènes humains : ils ont un « sens » et le sens, çà ne s'explique pas, çà se " comprend ». C'est à cette compréhension que je voudrais vous amener, en parlant de "la maternité et du sacré». Mais une précision d'abord: je ne voudrais surtout pas opposer les explications scientifiques et techniques à la compréhension du sens, l'expérience médicale à l'expérience du sacré. Plus les sciences et techniques, en effet, expliquent les mécanismes du développement de la vie, plus elles soulignent la complexité de cette vie et suscitent notre étonnement et notre admiration; Autrement dit, l'explication nous aide à mieux comprendre le sens mystérieux de la vie. J'examinerai d'abord la dimension symbolique du sacré dont témoignent des expressions bien connues qui soulignent le rôle éminent de la mère, puis la dimension tutélaire du sacré fondée sur la dyade "mère-enfant » et ritualisée dans les soins qui précèdent et suivent l'accouchement, enfin, ce que j'appellerai la dimension éthique du sacré qui greffe l'expérience de la maternité sur celle de la paternité.

La dimension symbolique du sacré Les attestations du langage

3 Je commencerai tout bonnement par déchiffrer ce sens en laissant résonner en nous l'écho de mots qui nous sont familiers .D'abord, «pro-créer» : cela veut dire créer à la place de, au lieu de..., co-créer, ce qui signifie que l'homme et la femme deviennent créateurs à la place (pro) du Créateur, de Dieu, créateurs avec (co) avec lui... Il y a aussi le terme " engendrer " qui dit la même chose, mais autrement. Engendrer, c'est être à l'origine d'un phénomène, c'est causer, produire, en un sens naturel et biologique 
bien sûr, mais aussi en un sens symbolique : c'est générer de l'humain , c'est faire entrer un être dans le genre humain (in-genere), c'est faire preuve de générosité . Générer, c'est être généreux : c'est donner ou transmettre ce que l'on a déjà reçu, avec tous les aléas que comporte un tel geste. L'expression «donner la vie» est encore la plus belle et la plus significative pour désigner le sacré de la maternité et de la paternité .Donner la vie, c'est faire l'expérience de l'être qui nous vient d'ailleurs, d'un don vécu dans une chaîne de transmission inter-générationnelle. Il y a aussi la belle expression "mettre quelqu'un au monde ». Enfanter, c'est faire en sorte que quelqu'un d'autre partage notre monde, tisse ce monde avec les mots qu'on lui offre, ceux de sa langue maternelle, qu'il " voie le jour", habite la "clairière » de la vie et se meuve dans la même forêt de symboles. Mais ce travail de procréation, d'engendrement, de don de la vie et de mise au monde commence dès la grossesse : il est le temps de la gestation de l'élaboration, du développement des potentialités biologiques, affectives et mentales, de l'éveil du désir et de la structuration des premières couches de l'inconscient, lieu de ressourcement de soi et de santé psychique.

Mais bien sûr, le sens de ces quatre termes se recoupe dans le mot " concevoir » qui signifie bien entendu, du point de vue du couple, former un enfant par le rapprochement sexuel, mais aussi, du point de vue de la femme, le former en soi : le "concevoir», c'est, au sens intellectuel et affectif, se représenter l'enfant par anticipation, le rêver, le désirer. Le bébé est sans doute d'abord fantasmé comme étant l'enfant idéal, l'enfant "trophée ", mais peu à peu, il devient l'enfant bien réel, bien ordinaire : notre fils, notre fille. Le terme « concevoir " recouvre aussi tout le travail que la future mère fait sur elle-même, pour réduire l'écart existant entre le rêve excessif dont est "auréolé » l'enfant et la réalité à venir, non encore manifestée : concevoir, c'est finalement se préparer à accueillir l'enfant, tel qu'il est, cet enfant qui, paradoxalement, est bien ici, mais pas encore là, étant « en sursis d'être -au monde »...

Tous ces termes nous renvoient à l'expérience du sacré : les parents et, d'une façon particulière la mère, sont comme happés par l'Inconnu qui exerce sur eux fascination et angoisse, crainte et séduction, mais ils sont du même coup mis sous la dépendance de cet Inconnu et donc comme dépassés par l'événement qu'ils vivent. Ils se sont risqués à concevoir de l'inconcevable... C'est sans doute cette expérience du sacré qui s'exprime de façon particulière dans le questionnement vertigineux qui, de temps à autre, traverse l'esprit et le cœur de la future mère : comment peut-on donner la vie à un être qui est par définition mortel?; pourquoi cet enfant qui est si proche de moi m'échappe-t-il déjà et devra-t-il faire un jour sa vie sans moi ? pourquoi est-il aussi présent et me laisse-t-il aussi seule ?; pourquoi, en donnant la vie, je risque aussi de "donner " la mort ?; pourquoi ce bonheur d'enfanter est-il aussi inséparable de l' angoisse de l'imprévu ? On le comprend, ces questions radicales que se pose la future mère butent sur les limites de l'énigme de la vie humaine. Ces questions, elle se les pose dans une solitude singulière, comme l'a indiqué Françoise Dolto dans son beau livre « Solitude $»^{1}$.

On le voit, l'expérience du sacré commence, dès lors que l'on ne réduit pas les gestes humains aux mécanismes biologiques, mais qu'on les considère dans leur portée symbolique, en respectant le sens et les valeurs qu'ils comportent. Telle est la première dimension du sacré que l'on peut qualifier de symbolique. Certes, la maternité implique bien entendu cette dimension, mais elle témoigne plus radicalement du sacré dans la mesure où les gestes qui l'expriment sont des gestes fondateurs qui engagent le 
désir de la mère et celui de l'enfant. Ce sacré que je qualifierai de mythique , car il comporte, Dieu merci, une bonne part d'imaginaire fondateur, mais aussi de mystique , car il inaugure entre la mère et l'enfant un lien des plus forts qui soient rassemble tous les éléments qui définissent cette expérience: relation de dépendance et d'interdépendance de l'enfant et de la mère, relation fondée sur une communion inédite faite d'absence et de présence durant la grossesse, enfin relation qui pousse au paroxysme les sentiments ambivalents de crainte et de séduction (fascinans et tremendum), comme dirait Otto, un des théoriciens le plus connu du Sacré.

On peut enfin souligner que cette dimension symbolique du sacré qui fait entrer l'enfant dans une communauté culmine avec l'imposition du nom à l'enfant. C'est l'enfant qui, une fois nommé, devient sacré, car il se trouve « distingué » des autres, mis à part. Il occupe donc une place singulière, il devient quelqu'un. Dans la Bible, le Créateur nomme les êtres qu'il crée et, ce faisant, il les met à part, leur confère une dimension sacrée.

\section{Le désir de la mère}

La relation est mise en œuvre du désir et le désir est mise en œuvre de la relation. Comment comprendre cette articulation du désir et de la relation dans la maternité ? On peut, me semble-t-il, répondre à cette question, en indiquant que la maternité implique une liaison étroite entre féminité, fécondité et sexualité. La maternité accomplit la féminité ${ }^{2}$. J. Lacan voit dans ce qu'il appelle la Femme le paradigme du désir ouvert, tissé d'une attente active et orienté vers l'Inconnu. On pourrait dire que la Femme- et il faut préciser que l'homme n'échappe pas à cette loi du désir dont toute femme témoigne d'une façon radicale et spécifique- symbolise l'énigme du désir humain. Ce qui fascine dans le désir, c'est son caractère énigmatique. Le désir ne s'appuie sur aucune raison, et, sauf à se renier, il ne saurait se satisfaire d'aucun accomplissement, même de cet accomplissement que serait la naissance d'un enfant trop désiré. Il est, du début à la fin, fondamentalement gracieux dans sa fragilité et fondamentalement généreux, car il nous fait être, nous génère et nous régénère, toujours ouvert sur l'avenir. La féminité exprime l'énigme du désir, mais cette énigme nous renvoie à la maternité .Pensons au sourire énigmatique de la Joconde, qui exprime, sur le mode d'une jouissance contenue, le lien entre féminité et maternité : Mona Lisa dont Léonard de Vinci n'a peint que le buste était, on le sait, enceinte.

Le lien secret existant entre la féminité et la maternité, c'est la fécondité .Or, la fécondité fait partie essentielle de la sexualité féminine. C'est sur ce point que l'on mesure la timidité et l'insuffisance des analyses de Freud sur la sexualité de la femme qui est toujours analysée en contraste avec la sexualité masculine. Certes, il ne s'agit pas de réduire pour autant la sexualité féminine à cette dimension, si essentielle soielle. On aurait d'ailleurs raison de se méfier de certaines jeunes filles qui voudraient tout suite se marier pour faire des enfants. Le désir comme expression de la fécondité suppose maturation et maturité et, par ailleurs, il est subordonné à la rencontre avec un homme qui ne soit pas qu'un partenaire sexuel d'occasion, mais qui soit capable de « répondre » à ce désir et de s'en porter garant .La fécondité n'est donc pas qu'une sublimation du désir sexuel, au sens freudien: elle en est l'accomplissement, mais l'accomplissement différé jusqu'à la rencontre de deux désirs

11 Or, ce sont ces deux caractéristiques du désir qui vont se réaliser en se croisant dans l'expérience de la maternité .Bien sûr, il paraît un peu téméraire de disserter sur la 
maternité, quand on est un homme. Mais je dirai qu'il n'est pas toujours évident, et les psychologues et les psychanalystes le savent bien, de rendre compte d'un vécu, dans lequel nous sommes très fortement impliqués... On répète à l'envi qu'il faut en être passé par là, par la maladie, par l'amour, par la maternité etc., pour comprendre ce dont on parle... Certes, l'expérience est essentielle, mais quand on la vit et même quand on l'a vécue, il n'est pas toujours facile de prendre le recul nécessaire pour en rendre compte et trouver les mots justes pour l'exprimer. On peut cependant parler, plus aisément, d'une expérience que l'on a croisée, soit parce que on l'a vécue par sympathie et analogie: chacun de nous a été enfant et peut comprendre, en analysant son histoire ce qu'est l'attitude d'une mère qui l'a, au sens propre et figuré, " élevé »; soit parce qu'on l'a vécue de manière indirecte, comme dans l'expérience de la paternité qui prolonge et accomplit la maternité.

La maternité désigne le fait de mettre un enfant au monde et, par conséquent, recouvre la période prénatale de la grossesse, celle de la vie souterraine du fœtus, mais elle s'étend aussi à la période qui suit la naissance, celle de la vie du nouveau-né et du nourrisson, et, on s'en doute, à la période de la petite enfance qui va bien au-delà du sevrage. Comme je l'ai dit au début, mettre un enfant au monde, c'est accouché d'un enfant, mais c'est aussi guider ses premiers pas dans le monde, où il est comme jeté, dès le premier jour de sa naissance. Ainsi, la maternité recouvre-t-elle deux types d'accompagnement: celui, prénatal, où la mère vit en symbiose avec l'enfant, celui, post-natal, où la mère assiste l'enfant qui, sans les soins qu'elle lui prodigue, ne saurait survivre. Ces deux phases de la maternité commandent deux postures différentes du corps de la mère par rapport à celui de l'enfant .Durant la grossesse, c'est la mère qui se prolonge dans le corps de l'enfant et, et une fois venu au monde, c'est l'enfant qui se prolonge, pour un temps, dans le corps de la mère.

Le sacré tutélaire La dyade structurante

J'en viens à l'expérience du sacré qui spécifie la relation fusionnelle de la mère et de l'enfant. On dit souvent que la mère et l'enfant ne font qu'un. Cela signifie qu'il y a la mère, l'enfant et le «nous " qui les unit. C'est cette relation de communion quasimythique et mystique que l'on appelle la «dyade ", mais, on le voit, cette dyade ne saurait être exclusivement une relation duelle ou une relation en miroir puisque l'image de chacun échappe à l'autre, et pour cause. En clair, la dyade n'est pas refermée sur elle-même, puisqu'elle est ouverte à un "nous » qu'elle génère. On le devine, la mère étant le corps-réceptacle de l'enfant est à la fois le lieu d'un double indispensable à la survie de l'enfant, mais aussi déjà le lien potentiel incontournable avec le monde extérieur. Françoise Dolto a développé avec une rare finesse et délicatesse cette fonction transitionnelle de la mère: «Le bébé une fois séparé physiquement de sa mère, il faut que l'espace soit "mamaïsé »: toute ambiance peut avoir couleur de maman, toute odeur doit être référée un peu à celle de maman, même absente, tout peut être nimbé de maman $»^{3}$. Les psychanalystes dont Freud lui-même et après lui J. Lacan ont parlé de grand "Autre»: ce qui signifie qu'elle est à la fois une autre personne que l'enfant, mais aussi l' "Autre ", le tiers qui fait le lien entre l'intérieur et l'extérieur, le dehors et le dedans. En clair, la maternité n'est ni plus ni moins que l'exercice de cette double fonction à laquelle le père donnera un écho amplifié et conférera une portée sociale plus large. On le conçoit dès lors aisément : la fonction maternelle implique la fonction paternelle. 
D'une façon plus précise, on se demandera quels sont les caractères du sacré inhérents à cette fonction. Je dirai, d'abord, que le sacré est dans le rapport de communion ineffable, d'interdépendance mutuelle qui lie l'enfant et la mère. C'est par cette communion que vont se tisser et se construire, dès la grossesse, les premiers éléments rythmiques de l'image basique du corps de l'enfant, à partir des sonorités verbales, des bruitages corporels, des mouvements et des déplacements de la mère et que cette image basique est essentielle : elle a pour fonction d'animer le frêle schéma corporel du bébé embryonnaire. Mais, à côté de ce caractère primordial de relation communionnelle, nous rencontrons les deux autres traits caractéristiques du sacré : la fascination et l'angoisse. La fascination, c'est le sentiment de participer à un événement qui nous dépasse : celui de donner la vie que l'on a soi-même reçue et de participer, au jour le jour, à cette croissance de l'être en soi-même, d'en être comme le tabernacle. Mais l'angoisse, c'est celle de ne pas parvenir à voir l'être qui nous habite, de vivre cette présence de l'autre en soi déjà sur fond d'absence et de séparation, de sentir que cet être est un paradoxe d'étrangeté et de familiarité, qu'il est proche et lointain, de pressentir aussi que cette communion débouche sur l'Inconnu, de penser enfin que cette vie naissante inclut déjà la mort dans le processus même de son développement naturel.

Il convient de bien comprendre que cette expérience du sacré est fondatrice : elle est aussi bien essentielle à la mère qu'à l'enfant. La communion dont il s'agit implique une osmose permanente entre l'enfant et la mère. Or, c'est cette osmose qui contribue à asseoir les premières bases du narcissisme de l'enfant : le besoin de sécurité, de chaleur et de douceur définit le "sentiment océanique " qui est au principe du sacré et au principe de l'expérience de soi: «Les choses sont d'abord humanisées, déifiées... mamaïsées, la mère étant le Dieu de l'enfant, puisqu'elle semble régner sur tout. C'est quand l'enfant s'aperçoit que la mère, elle aussi, a été faite par quelqu'un d'autre, qu'alors elle n'est pas Dieu $»^{4}$. Mais il faut aussi dire que cet échange insolite avec la mère qui narcissise l'enfant contribue aussi à renarcissiser la mère, c'est-à-dire à modifier en profondeur le rapport qu'elle entretient vis-à-vis d'elle-même : la mère se positionne comme femme, mais autrement, différemment .L'enfant se constitue par la mère et la mère se reconstitue par l'enfant. La maternité restructure la féminité, en exauçant le désir de fécondité.

Mais l'expérience du sacré n'est pas seulement fondée sur des sentiments, si forts soient-ils : elle se réalise à travers tout un ensemble d'interventions, de soins, de régimes et de traitements dont vous rendez compte, dans ce congrès, de la nécessité avec une grande précision scientifique et technique. Mais ces gestes dont vous faites état et qui accompagnent la grossesse, vous le savez bien, ne sont pas que médicaux ou diététiques : ce sont des rites et des rituels qui viennent exprimer, entourer, renforcer et rythmer l'expérience du sacré.

Le rituel des soins

Je serai, en effet, tenté d'interpréter en termes de rituels les comportements nouveaux que la grossesse suscite chez la mère. Bien sûr, étant habité par un autre, elle ne saurait vivre de la même façon qu'auparavant : c'est alors que s'instaure entre la mère et l'enfant un dialogue insolite qui donne corps à cette expérience inédite du sacré. Mais font aussi partie de ces rituels le régime de vie de la mère : travail, détente, diététique et aussi - pourquoi pas? - les diverses démarches de soins : consultations gynécologiques, échographie, séances de préparation à l'accouchement... 
19 C'est bien entendu sur ce terrain rituel que se situe l'intervention des sages-femmes... Les gestes qui précèdent l'accouchement, les gestes qui l'accompagnent et ceux qui le suivent, si techniques soient-ils, ne sont pas seulement des actions mécaniques : ce sont bien des gestes, donc des démarches symboliques qui inscrivent le nouveau- né dans la relation sociale. Que l'on songe aux ébauches de rituels qui entourent l'accouchement et qui placent la sage-femme dans une fonction essentielle de médiation : accueil du père lors de l'accouchement, pesée et toilette du bébé, remise de ce dernier à sa mère et à son père... On peut observer d'ailleurs que ces rituels varient d'une culture à l'autre et que, comme cela a déjà été étudié, les femmes maghrébines, par exemple, se réfèrent, malgré la similitude des gestes, à des rituels différents, puisque la place de la mère dans la famille est essentielle ${ }^{5}$. On le voit, le rôle de la sage-femme n'est pas seulement d'ordre médical ou encore pédagogique : par ses interventions, mais aussi par ses paroles, elle a une fonction de médiation indispensable, puisqu'elle inscrit les premières expériences sociales de l'enfant dans l'espace transitionnel qu'elle ouvre et qui n'est autre que le tracé et le sillage du corps de la mère.

Une fois l'enfant mis au monde, la mère, en prodiguant les soins nécessaires à la survie de l'enfant (nourriture, toilettes, soins du corps), ne cesse de poser des gestes rituels. Elle assigne ainsi à l'enfant, par sa présence vigilante, un périmètre de sécurité qui, d'une certaine façon, est sacré, puisqu'il est exclusivement construit par la mère pour l'enfant. Elle est la «maman trompe-la-mort » qui rejette à l'extérieur ces intrus que est l'angoisse, la souffrance et la violence. Elle « esthétise » pour ainsi dire, valorise le moindre geste de l'enfant par son sourire, ses caresses et ses paroles : « Le beau c'est la quintessence de l'humain, ce sentiment précoce qui est lié à l'odeur du corps de la mère,à l'éclat de son regard -pour les enfants voyants- à la caresse auditive de la voix de la mère -pour ceux qui ne sont pas sourds- et à la tactilité spéciale reconnue de la mère, qui s'est reconnue en son enfant extérieur à elle $»^{6}$. La mère est le mot de passe de la beauté 7 . Bref, l'enfant trouve dans la présence de la mère l'enracinement d'un mode primordial d'être, de sentir, de réagir.

C'est en s'identifiant à la mère que l'enfant se construit. Mais cette identification à la mère " gestante » et «allaitante » ne se réalise jamais sans faille. .La mère tutélaire ne saurait être toujours là : si elle existe pour l'enfant, elle n'existe pas que par l'enfant. .L'expérience du sacré est faite de présence et d'absence. On le pressent, elle est la condition de l'éveil à la relation : les absences trop fréquentes de la mère qui font pleurer le bébé lui permettent de se projeter dans une image de soi dynamique et cette nouvelle image de soi vient reconfigurer l'image basique de son corps constituée par les premières identifications à la mère .C'est lorsqu'il est en attente que l'enfant passe du besoin au désir par le biais de la demande et cherche des substituts symboliques à la mère absente. On comprend que l'expérience du sacré soit faite de communion et de séparation. C'est par ces absences répétées et obligées que la mère pose les premiers interdits qui empiètent déjà sur la fonction paternelle qu'elle convie par le fait même. On pourrait dire que l'expérience du sacré débouche sur l'expérience du religieux, entendu au sens premier de ce qui lie et relie aux autres et à soi. De lieu qu'elle était, la mère se fait lien social...C'est par ce sevrage réel et symbolique que le nourrisson passe du besoin au désir et se construit en inventant les substituts symboliques qui vont convertir son attente passive en une attente active et le pousser à entrer en apprentissage, de s'inventer lui-même en babillant ou gesticulant ... Ce que l'on appelle le stade du miroir est le moment où l'enfant va faire la distinction entre son 
propre corps et celui de la mère et où il va refouler l'image de son corps, pour pouvoir se figurer et se projeter vers l'inconnu.

Mais parmi ces rituels, il en est un qui est privilégié et qui relève de l'apprentissage du langage. La langue, c'est bien la langue maternelle. Elle commence, on le sait, à se tisser durant la période de la grossesse : l'enfant entend les mots prononcés par la mère, mais aussi par le père, comme une musique; il les perçoit comme des caresses sonores, s'inscrit dans ce rythme de sonorités et de silences qui lui vient de l'ailleurs qu'il habite déjà. Si sédentaire soit-il, le futur bébé nomadise déjà dans le ventre maternel. C'est en écho à ce fond signifiant premier et aussi en écho aux paroles actuelles de la mère que l'enfant va émettre ces vocalisations spontanées que l'on appelle le babil et qu'il va prononcer peu à peu les premiers mots et se risquer un jour à parler. La mère enseigne la musique de la langue et les premières règles du langage.

\section{La dimension éthique du sacré paternel}

Le sacré tutélaire qui est fondé sur la communion de la mère et de l'enfant débouche sur une troisième dimension du sacré: celle qui est fondée sur la reconnaissance de l'enfant par la mère. Pour recourir à un vocabulaire qui est plus tranché, je dirai que le sacré "mystique" de la maternité se prolonge dans un "sacré » éthique qui exige le respect de l'enfant comme personne humaine. D'une certaine façon, cette nouvelle dimension qui découle de la première introduit, dans la communion, une distance que la paternité a pour fonction d'expliciter et de renforcer... Si intime et si intense soit le rapport de communion de la mère à l'enfant, au point que l'on puisse le qualifier d'ineffable, il doit inclure la reconnaissance de l'enfant comme autre, comme être différent, comme être singulier. L'enfant est le premier " prochain " à aimer et à respecter. Il n'est pas une excroissance du corps de la mère : le cordon ombilical réel et imaginaire doit être coupé pour laisser place au lien symbolique du respect. Il nous faut faire quelques remarques préalables, de type historique, pour mieux apprécier l'enjeu de cette expérience.

1) Cette conception philosophique et socio-politique de l'enfant ne va pas de soi. Elle est une conquête du judéo-christianisme, de la raison philosophique et de la démocratie. Dans le droit romain, l'enfant est un bien, une possession, une richesse, une " jouissance ", au sens juridique du terme. L'enfant est une propriété des parents. On « a » des enfants ou on n'en «a » pas. Cette revendication de l' « avoir » n'est certes pas absent de certaines mentalités: l'expression courante: vous «avez» combien d'enfants? Nous renvoie encore en écho à cette signification, quand bien même le verbe "avoir " n'a plus la signification de " posséder », au sens strict et juridique du terme. En fait, ces expressions expriment, mais de façon paradoxale, autre chose: avoir des enfants, cela fait partie de la dimension de l'être des parents, de sorte que celui, celle ou ceux qui n'ont pas d'enfant pourraient être considérés comme des êtres non totalement accomplis, comme s'il leur manquait une dimension essentielle.

2) Mais laissons ces considérations qui pourraient nous entraîner encore plus loin. Avec le christianisme, la dimension de l'avoir s'est estompée au profit de la dimension de l'être. Rappelons-nous le célèbre jugement de Salomon qui, devant la revendication de deux mères, propose de partager l'enfant en deux comme s'il s'agissait d'un bien ou d'une marchandise .Jugement tranchant qui marque la différence entre avoir et être : la vraie mère se manifeste aussitôt: elle proteste de façon véhémente et préfère n' « avoir» pas l'enfant, car, au moins, l'enfant continuera d' «être», quand bien 
même elle ne l' " aura " plus. Mais cette valorisation de l'être au détriment de l'avoir qui change, on s'en doute, du tout au tout la vision sociale de l'enfant ne s'est pas pour autant imposée d'emblée dans la culture avec toutes les conséquences qu'elle implique .L'enfant qui reste un être au sens d'une créature, donc d'un être imparfait et dépendant est encore considéré, durant la période médiévale, comme un être humain en miniature, amoindri, rebelle, fragile et inconscient, imprévisible, voire un tantinet pervers. Ce n'est que peu à peu, à partir du $17^{\text {ème }}$ et surtout du $18^{\text {ème }}$ siècle- que l'on pense au roman éducatif "Emile» que Rousseau publie en 1762- que l'enfant- créature devient l'enfant-sujet et, aujourd'hui, sujet de droits et de devoir, à l'instar de toute personne humaine. Ce qui signifie que l'enfant est un être singulier, "quelqu'un », un individu et une personne. Comme sujet, il n'appartient pas aux parents : il appartient à soi-même, il s'appartient...

3) De ce point de vue, l'enfant est, en tant que personne humaine, un être sacrer que l'on ne saurait s'aliéner, s'approprier ou s'asservir de quelque façon que ce soit. Le sacré est ici fondé sur le respect dû au bébé en raison même de sa vulnérabilité. Il doit être reconnu comme tel, comme personne et sujet à part entière. Le respect s'adresse à l'enfant tel qu'il est et non tel qu'il devrait être. Respecter quelqu'un, c'est bien le regarder et au besoin corriger le regard que l'on a posé sur lui (le terme respect vient du latin « re-aspicere : y regarder à deux fois), le voir au-delà des apparences, audelà de l'aspect physique, psychologique ou moral qu'il présente (là, on n'y regarde qu'une seule fois et on juge). Avec cette conception de l'enfant-sujet, le sacré de communion se transmue en sacré de référence, le sacré tutélaire en sacré «éthique ». Bref, le sacré de la maternité inaugure le sacré de la paternité : ces deux dimensions du sacré sont complémentaires. La dyade tutélaire risque, en effet, de se refermer sur une sacralisation mythique, étouffante et mortifère si elle ne s'ouvre pas à la parole paternelle qui transforme la dyade inauguratrice "mère-bébé» en une trinité fondatrice. En réalité, on ne saurait séparer le sacré maternel de type tutélaire du sacré paternel de type éthique et symbolique, puisque le père est « l'actif initiateur du sens à l'agir $»^{8}$. Nous avons toutes et tous présents à l'esprit ces tableaux mièvres de la crèche où l'enfant divin se trouve au centre, entouré de femmes vêtues de dentelles avec tout autour les bergers et les animaux et, quasiment à l'extérieur ou en retrait du cadre, un vieillard chauve qui ne peut être que le père. Cette maternité généreuse à laquelle la nature est associée est l'image du sacré mystique d'une dyade d'où serait plus ou moins exclu le sacré de la parole paternelle.

Je voudrais conclure en disant un mot de la place de la sage-femme dans ce dispositif. La sage-femme assure, me semble-t-il, la transition ou la médiation entre l'enfant rêvé et imaginé et l'enfant tel qu'il est, et hélas, parfois malade, transféré, différent, abandonné, décédé...Comme la mère, la sage-femme esthétise en quelque sorte le choc de la réalité et présente l'enfant à sa mère, ainsi qu'à son père .A sa façon, elle se situe dans le prolongement du sacré tutélaire de la dyade et dans le sillage du sacré éthique de la paternité. Elle confère, en effet, aux gestes médicaux une signification rituelle en séparant l'enfant de la mère, en participant au triomphe de la vie sur la mort, en s'enquérant du nom du bébé pour le nommer à son tour, en le soumettant aux rituels de la toilette et de la pesée, en le remettant à sa mère et aussi à son père. La sagefemme entoure finalement la naissance "par un cadre et des relations 
complémentaires à ceux qu'exige la médecine $»^{9}$ et est habilitée à "faire participer le père $»^{10}$ à l'événement de la naissance. Ainsi est-elle amenée, par cette subtile «maïeutique » physique et spirituelle qui fait tout son art, à jouer elle aussi le rôle d'un tiers et à témoigner de l'énigme, du mystère de la maternité et de la paternité. Mais aussi, elle peut, à sa façon, mettre en perspective la dimension spirituelle de l'événement, en signalant la naissance par quelques signes (Wincke), par quelques gestes symboliques, en faisant place, dès les visites à la maternité aux rituels familiaux ${ }^{11}$, puis, en préparant le retour festif à la maison.

Schiltigheim le 26 Mars 2006

Conférence prononcée à l'occasion des $34^{\text {ième }}$ assises nationales des sages-femmes

(17 $7^{\text {ième }}$ session européenne), Strasbourg, 10, 11, 12 mai 2006

\section{NOTES}

1. F. Dolto, « Solitude », Paris, Folio/ essais, 1994

2. E. Lemoine-Luccioni, « Grossesse et Féminité » in Partage de femmes, Paris, Seuil, 1976, p. 31sq.

3. F. Dolto, op. cit. p. 29

4. F. Dolto, op. cit. p. 76

5. Mohamed Belhadj, « Les rites et l'espace maternel traditionnels maghrébins » in Maternité et sacré, Spirale $n^{\circ} 2,1926$, p. 27sq

6. F. Dolto, op. cit. p. 59

7. F. Dolto, op. cit. p. 60

8. F. Dolto, op. cit. p. 149

9. O. de Dinechin, «L'approche chrétienne traditionnelle et son actualisation », in Maternité et sacré, op. cit. p 43

10. Ibidem

11. Ibidem

\section{RÉSUMÉS}

Dans ce texte qui est le compte-rendu d'une conférence prononcée à Strasbourg dans le cadre des 34èmes Assises nationales des Sages-femmes (les 10,11, 12 Mai 2006), l'auteur insiste sur la dimension symbolique des soins que prodigue la mère à son nouveau-né et que les sages-femmes doivent assurer et garantir. Cette dimension implique une expérience du sacré attestée par le langage (pro-créer, engendrer, mettre au monde...), par les modes d'investissement du désir de la 
mère vis-à-vis de son enfant et, bien entendu, par le rituel social des soins qui ne sauraient être réduits à leur dimension technique et physique.

\section{AUTEUR}

JEAN-PAUL RESWEBER

Pr. Université Paul Verlaine (Metz) 